\title{
Evaluation of the target genes of arsenic trioxide in pancreatic cancer by bioinformatics analysis
}

\author{
CONG-YA ZHOU ${ }^{1}$, LIU-YUN GONG ${ }^{1}$, RONG LIAO ${ }^{2}$, NING-NA WENG ${ }^{2}$, YAO-YUE FENG ${ }^{2}$, YI-PING DONG ${ }^{1}$, \\ HONG ZHU ${ }^{2}$, YA-QIN ZHAO ${ }^{2}$, YUAN-YUAN ZHANG ${ }^{1}$, QING ZHU ${ }^{2}$ and SU-XIA HAN ${ }^{1}$ \\ ${ }^{1}$ Department of Oncology, The First Affiliated Hospital, Xi'an Jiaotong University, Xi'an, Shaanxi 710061; \\ ${ }^{2}$ Department of Abdominal Oncology, West China Hospital of Sichuan University, Chengdu, Sichuan 610041, P.R. China
}

Received January 14, 2019; Accepted August 7, 2019

DOI: $10.3892 / \mathrm{ol} .2019 .10889$

\begin{abstract}
The aim of the present study was to evaluate the potential network of arsenic trioxide (ATO) target genes in pancreatic cancer. The DrugBank, STITCH, cBioPortal, Kaplan-Meier plotter and Oncomine websites were used to analyze the association of ATO and its target genes with pancreatic cancer. Initially, 19 ATO target genes were identified, along with their associated protein-protein interaction networks and Kyoto Encyclopedia of Genes and Genomes pathways. ATO was found to be associated with multiple types of cancer, and the most common solid cancer was pancreatic cancer. A total of 6 ATO target genes (namely AKT1, CCND1, CDKN2A, IKBKB, MAPK1 and MAPK3) were found to be associated with pancreatic cancer. Next, the mutation information of the 6 ATO target genes in pancreatic cancer was collected. A total of 20 ATO interacting genes were identified, which were mainly involved in hepatitis B, prostate cancer, pathways in cancer, glioma and chronic myeloid leukemia. Finally, the genes CCND1 and MAPK1 were detected to be prognostic factors in patients with pancreatic cancer. In conclusion, bioinformatics analysis may help elucidate the molecular mechanisms underlying the involvement of ATO in pancreatic cancer, enabling more effective treatment of this disease.
\end{abstract}

Correspondence to: Dr Su-Xia Han, Department of Oncology, The First Affiliated Hospital, Xi'an Jiaotong University, 277 Yanta West Road, Xi'an, Shaanxi 710061, P.R. China

E-mail: shan87@mail.xjtu.edu.cn

Dr Qing Zhu, Department of Abdominal Oncology, West China Hospital of Sichuan University, 37 Guoxue Lane, Chengdu, Sichuan 610041, P.R. China

E-mail: newzhuqing1972@yahoo.com

Abbreviations: ATO, arsenic trioxide; PPI, protein-protein interaction; KEGG, Kyoto Encyclopedia of Genes and Genomes

Key words: arsenic trioxide, pancreatic cancer, bioinformatics analysis, Kyoto Encyclopedia of Genes and Genomes pathway, interacting genes

\section{Introduction}

Pancreatic cancer is one of the most aggressive malignancies worldwide, with a mortality rate that is nearly equal to its incidence rate (1). Pancreatic cancer is expected to become the second leading cause of cancer-associated mortality in the US by 2030 (2). Despite advances in diagnostic and therapeutic strategies over the past years, the prognosis of patients with pancreatic cancer remains unsatisfactory, with a 5-year survival rate of $<8.5 \%$ (3). Pancreatic cancer has become one of the major public health concerns worldwide. In order to improve the treatment of pancreatic cancer, it is necessary to elucidate the pathological mechanism underlying its development, identify a drug-based framework and investigate the functional mechanisms involved.

Arsenic trioxide (ATO) has been reported to be an effective therapeutic agent for acute promyelocytic leukemia (APL) (4). It has been reported that ATO can induce molecular remission, as well as prolong the overall survival of patients with APL $(5,6)$. Combined treatment with retinoic acid (RA) and ATO has been demonstrated to be curative for the majority of patients with APL (7-9). Furthermore, an increasing number of studies have proven the anti-carcinogenic properties of ATO in different tumors, including in gastric cancer (10), lymphoma (11), bladder cancer (12), head and neck cancer (13), and ovarian cancer (14). Although it has been reported that ATO may inhibit the progression of pancreatic cancer (15), the potential targets and molecular mechanisms of action of ATO remain unclear.

In recent years, with the continuous improvement and development of high-throughput sequencing technology, multi-omics research (such as genomics, transcriptomics, proteomics and metabolomics) has gradually improved and has been used to identify disease-associated genes, which may uncover the molecular mechanisms underlying disease development and enable effective treatment. DrugBank is a richly annotated web-based bioinformatics tool that contains multiple drug data with comprehensive drug targets information (16). It provides detailed, step-by-step information on the results of previous studies on drugs, drug targets and drug effects, allowing researchers to search, view and export data, text and image (17). Furthermore, Search Tool for Interactions of Chemicals (STITCH) is an online database of the interaction networks of chemicals and proteins (18). 
In the present study, DrugBank and STITCH were used to identify the targets of ATO, in order to construct an ATO-target interaction network. Protein-protein interaction (PPI) network construction and Kyoto Encyclopedia of Genes and Genomes (KEGG) pathway analysis demonstrated that ATO is associated with several types of malignancies, including pancreatic cancer. In total, 6 genes (AKT1, CCND1, CDKN2A, IKBKB, MAPK1 and MAPK3) were identified as the target genes of ATO in pancreatic cancer. The interconnection of these 6 ATO target genes in pancreatic cancer was then analyzed using the Search Tool for the Retrieval of Interacting Genes (STRING) database. The cBioPortal website was further used to analyze the genomics data of these 6 target genes, while online Kaplan-Meier (K-M) plotter was used to analyze the prognosis of pancreatic cancer. In addition, the Oncomine website was used to reveal the expression levels of ATO target genes in pancreatic cancer tissues. Taken together, the findings of the bioinformatics analysis performed in the present study may help determine the gene targets of ATO and provide new therapeutic approaches for pancreatic cancer.

\section{Materials and methods}

Search for target genes of ATO. DrugBank, version 5.0 (https://www.drugbank.ca), is a richly annotated web-based bioinformatics tool that contains multiple drug data with comprehensive drug target information. In the present study, the DrugBank 5.0 and STITCH (http://stitch.embl.de/) databases were used to identify the ATO-target interactions in order to obtain an ATO-target network. The information on ATO target genes was then used to construct a visualization table and analyzed by STRING for further analysis. The search term 'arsenic trioxide' was used to search the DrugBank 5.0 and STITCH databases, and the targets of ATO in these two websites were identified. A total of 19 targets were recorded and included in subsequent analyses.

KEGG pathway and Gene Ontology analyses, and PPI network construction of ATO targets. The STRING database (https://string-db.org/) gathers and provides known and predicted PPI data for a large number of organisms, including humans $(19,20)$. Cytoscape is a general-purpose software platform used for integrating bimolecular interaction networks (21). CluePedia, a plugin of Cytoscape software, can be used for searching potential genes associated with the reported signaling pathway by calculating linear and non-linear statistical dependencies from experimental data (22). In the present study, the KEGG pathway and Gene Ontology (GO) analyses were conducted using the STRING database. Subsequently, the STRING website and Cytoscape software were utilized to establish a PPI network of the ATO target genes.

Genomics information of ATO targets in pancreatic cancer. The cBioPortal for Cancer Genomics (http://www.cbioportal. org/) is a free platform that is used for finding multidimensional cancer genomics data (23). OncoPrint is an online tool, which can visualize alterations of tumor samples from gene arrays. The alterations of 6 ATO target genes associated with pancreatic cancer (AKT1, CCND1, CDKN2A, IKBKB, MAPK1 and MAPK3) were visualized using cBioPortal and
Table I. Target genes $(n=19)$ of arsenic trioxide identified using DrugBank 5.0 and STITCH.

\begin{tabular}{ll}
\hline Target gene & \multicolumn{1}{c}{ Source } \\
\hline IKBKB & Drugbank 5.0 \\
TXNRD1 & Drugbank 5.0 \\
JUN & Drugbank 5.0 \\
CCND1 & Drugbank 5.0 \\
MAPK3 & Drugbank 5.0 \\
MAPK1 & Drugbank 5.0 \\
AKT1 & Drugbank 5.0 \\
CDKN1A & Drugbank 5.0 \\
PML & Drugbank 5.0 and STITCH \\
HDAC1 & STITCH \\
RARA & STITCH \\
ESR1 & STITCH \\
CDKN2A & STITCH \\
ABCC1 & STITCH \\
SP1 & STITCH \\
DNMT3B & STITCH \\
DNMT1 & STITCH \\
HSPA4 & STITCH \\
ABCB1 & STITCH \\
\hline
\end{tabular}

OncoPrint (24), and the genomic alteration frequency within the specific cancer study, listed below, was applied as a filter. In the present study, a total of 850 pancreatic cancer samples were analyzed in this section, which included International Cancer Genome Consortium (ICGC; $n=99$ ), Queensland Centre for Medical Genomics (QCMG2016; n=456), The Cancer Genome Atlas (TCGA; $\mathrm{n}=186$ ) and University of Texas Southwestern Medical Center (UTSW; n=109) datasets (25-28).

Overall survival analysis of ATO target genes in patients with pancreatic cancer. The K-M plotter (http://kmplot. com/analysis/) is a free online tool that can be used to assess the association of multiple genes with the survival of patients with cancer using different cancer samples (29). According to mRNA expression data from the aforementioned 6 ATO genes, the patients with pancreatic cancer were divided into the high- and low-expression groups based on the median expression value, and then K-M survival curves were constructed. In the present study, the information of 177 patients with pancreatic cancer was acquired from the K-M website, and the K-M survival curves were used to analyze the association between the expression of ATO target genes (AKT1, CCND1, CDKN2A, IKBKB, MAPK1 and MAPK3) and the prognosis of 177 patients with pancreatic cancer.

Determination of the expression of the 3 ATO target genes in pancreatic cancer. Oncomine (https://www.oncomine. org/) is an online cancer microarray website that facilitates the discovery of genome-wide expression analyses (30). In the present study, differential gene expression was detected by Oncomine when comparing cancer tissues with normal 
Table II. Kyoto Encyclopedia of Genes and Genomes pathways associated with the arsenic trioxide target genes.

\begin{tabular}{|c|c|c|c|}
\hline Pathway & Count & False discovery rate & Genes \\
\hline MicroRNAs in cancer & 10 & $5.09 \times 10^{-15}$ & $\begin{array}{l}\text { ABCB1, ABCC1, CCND1, CDKN1A, CDKN2A, DNMT1, } \\
\text { DNMT3B, HDAC1, IKBKB, MAPK1 }\end{array}$ \\
\hline Pathways in cancer & 11 & $7.73 \times 10^{-14}$ & $\begin{array}{l}\text { AKT1, CCND1, CDKN1A, CDKN2A, HDAC1, IKBKB, JUN, } \\
\text { MAPK1, MAPK3, PML, RARA }\end{array}$ \\
\hline Chronic myeloid leukemia & 8 & $7.73 \times 10^{-14}$ & $\begin{array}{l}\text { AKT1, CCND1, CDKN1A, CDKN2A, HDAC1, IKBKB, } \\
\text { MAPK1, MAPK }\end{array}$ \\
\hline Acute myeloid leukemia & 7 & $2.41 \times 10^{-12}$ & AKT1, CCND1, IKBKB, MAPK1, MAPK3, PML, RARA \\
\hline Pancreatic cancer & 6 & $6.88 \times 10^{-10}$ & AKT1, CCND1, CDKN2A, IKBKB, MAPK1, MAPK3 \\
\hline Glioma & 6 & $6.88 \times 10^{-10}$ & AKT1, CCND1, CDKN1A, CDKN2A, MAPK1, MAPK3 \\
\hline Hepatitis B & 7 & $1.11 \times 10^{-9}$ & AKT1, CCND1, CDKN1A, IKBKB, JUN, MAPK1, MAPK3 \\
\hline Melanoma & 6 & $1.11 \times 10^{-9}$ & AKT1, CCND1, CDKN1A, CDKN2A, MAPK1, MAPK3 \\
\hline Prostate cancer & 6 & $3.56 \times 10^{-9}$ & AKT1, CCND1, CDKN1A, IKBKB, MAPK1, MAPK3 \\
\hline Viral carcinogenesis & 7 & $4.54 \times 10^{-9}$ & $\begin{array}{l}\text { CCND1, CDKN1A, CDKN2A, HDAC1, JUN, MAPK1, } \\
\text { MAPK3 }\end{array}$ \\
\hline
\end{tabular}

pancreatic tissues. The studies by Ishikawa et al (31), Iacobuzio-Donahue et al (32), Pei et al (33), Badea et al (34), Logdons et al (35) and Segara et al (36) were used to reveal the expression of 3 ATO target genes in pancreatic cancer. Differences in transcriptional expression were compared using the Student's t-test. $\mathrm{P}<0.01$ was used to indicate a statistically significant difference and fold change was set to 2 .

\section{Results}

Evaluation of ATO target genes. ATO is a chemotherapeutic agent of idiopathic function used to treat leukemia that is unresponsive to first-line agents. It is suspected that ATO induces cancer cells to undergo apoptosis (11). In the current study, DrugBank and STITCH tools were used to identify the target genes of ATO. As shown in Table I, a total of 19 target genes were identified, including IKBKB, TXNRD1, JUN, CCND1, MAPK3, MAPK1, AKT1, CDKN1A, HDAC1, PML, RARA, ESR1, CDKN2A, ABCC1, SP1, DNMT3B, DNMT1, HSPA4 and $\mathrm{ABCB} 1$.

Gene Ontology (GO) analysis. The online STRING software was used to determine functional enrichments based on 19 ATO target genes. GO analysis revealed a significant difference in biological processes, which included 'response to lipid', 'DNA-templated transcription', 'initiation, regulation of transcription from RNA polymerase II promoter', 'positive regulation of gene expression', and 'transcription initiation from RNA polymerase II promoter,' among others. The molecular function terms identified included 'enzyme binding', 'transcription factor binding', 'sequence-specific DNA binding', 'cyclin-dependent protein serine/threonine kinase regulator activity' and 'transcription corepressor activity', among others. As regards the area of the cell components, the 19 ATO target genes were enriched in 'chromatin', 'nucleoplasm', 'nuclear chromatin', 'chromosome' and 'nuclear lumen'. The top 5 terms for biological processes, molecular function and cellular components were shown in Table SI.
Association between ATO targets and pancreatic cancer. The PPI network (Fig. 1) and KEGG pathway analysis for the 19 ATO target genes (Table II) were obtained from the STRING database. The top 10 signaling pathways associated with the ATO target genes were as follows: MicroRNAs in cancer, pathways in cancer, chronic myeloid leukemia, acute myeloid leukemia, pancreatic cancer, glioma, melanoma, hepatitis B, pancreatic cancer and viral carcinogenesis. The most common solid tumor was pancreatic cancer $\left(\mathrm{P}=6.88 \times 10^{-10}\right)$. A total of 6 genes, namely AKT1, CCND1, CDKN2A, IKBKB, MAPK1 and MAPK3, were found to be associated with pancreatic cancer. Subsequently, CluePedia software was used to verify and visualize the association of ATO target genes with pancreatic cancer, and the results were consistent with the STRING analysis results (Fig. 2).

Genetic data of ATO target genes in pancreatic cancer. The genetic information of these 6 genes in patients with pancreatic cancer were subsequently evaluated using the cBioPortal. The genomic alterations and clinical expression characteristics of AKT1, CCND1, CDKN2A, IKBKB, MAPK1 and MAPK3 in the aforementioned datasets ICGC $(n=99)$, QCMG2016 $(n=456)$, TCGA $(n=186)$ and UTSW $(n=109)$ were investigated (25-28). As shown in Fig. 3, the alteration rate of the 6 genes in pancreatic cancer varied between 0 and $63 \%$. AKT1 exhibited an alteration rate between 0 and 18\%, CCDN1 had an alteration rate between 0 and $10 \%, \mathrm{CDKN} 2 \mathrm{~A}$ had an alteration between 0 and $42 \%$, IKBKB had an alteration of $0-7 \%$, MAPK1 had an alteration between 0 and $8 \%$, and MAPK3 had an alteration between 0 and $9 \%$. Furthermore, AKT1, IKBKB and MAPK1 exhibited amplification, deep deletion and missense mutations. The alterations of CDKN2A included deep deletion, truncating, missense, inframe mutations and other mutations. The alterations of CCND1 and MAPK3 included amplification.

Interconnection of the 6 ATO target genes in pancreatic cancer. STRING analysis performed in the current study revealed that 


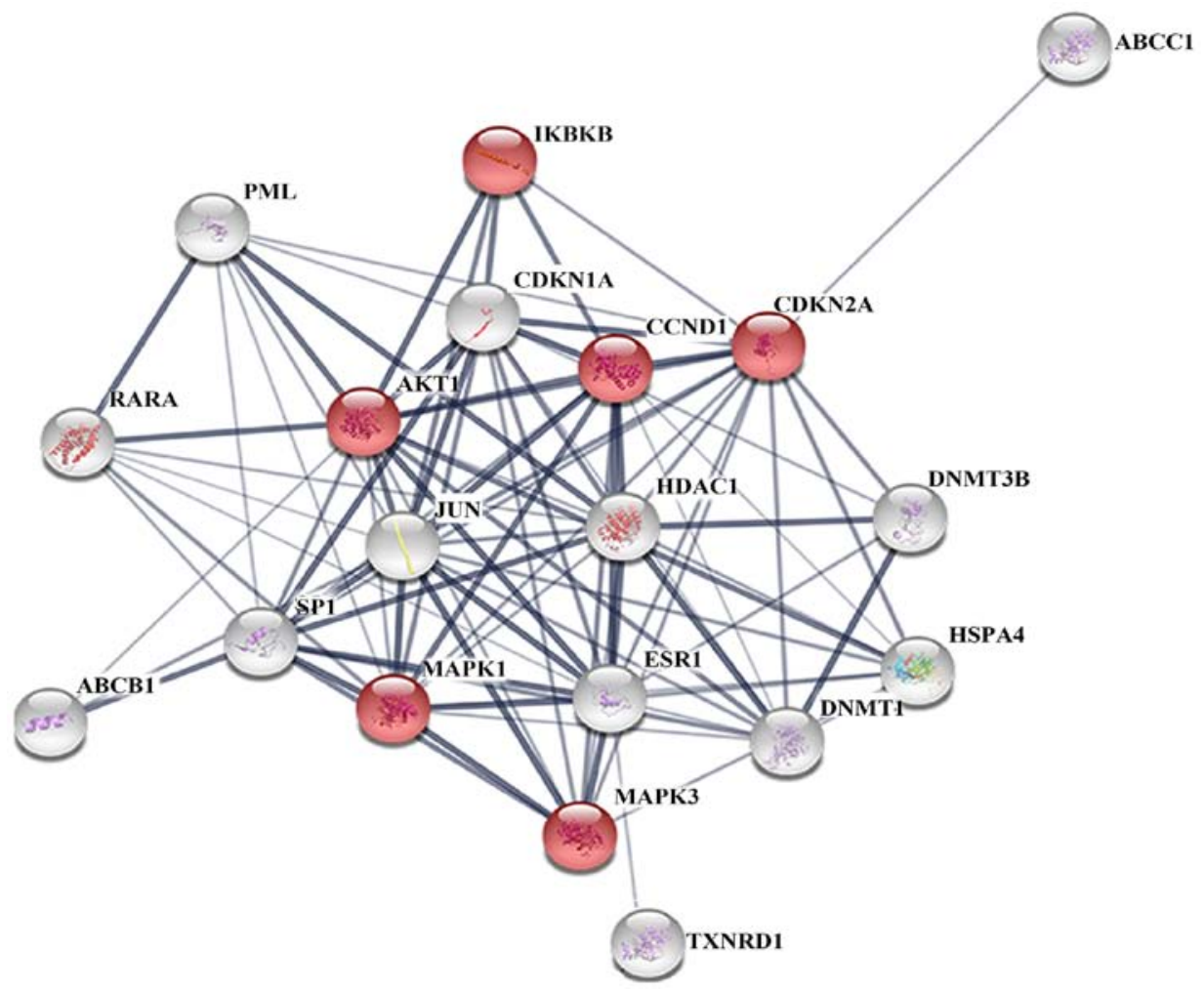

Figure 1. Protein-protein interaction network of arsenic trioxide target genes, including IKBKB, TXNRD1, JUN, CCND1, MAPK3, MAPK1, AKT1, CDKN1A, HDAC1, PML, RARA, ESR1, CDKN2A, ABCC1, SP1, DNMT3B, DNMT1, HSPA4 and ABCB1. Red color indicates association with pancreatic cancer.

the 6 ATO target genes (AKT1, CCND1, CDKN2A, IKBKB, MAPK1 and MAPK3) were involved in chronic myeloid leukemia, pancreatic cancer, non-small cell lung cancer, acute myeloid leukemia, melanoma, glioma, prostate cancer, endocrine resistance, FoxO signaling and hepatitis B (Table SII). A PPI network was constructed to analyze the genes interconnected with AKT1, CCND1, CDKN2A, IKBKB, MAPK1 and MAPK3. A total of 20 ATO interacting genes (FOXO1, CDK6, CDK4, RICTOR, IKBKG, FOXO3, PTEN, NFKB1, PIK3CB, JUN, TP53, PIK3CA, PIK3CG, CDKN1A, CDKN1B, CDK2, PIK3CD, MTOR, NFKBIA and RB1) were identified and were mainly involved in hepatitis $\mathrm{B}$, prostate cancer, pathways in cancer, glioma and chronic myeloid leukemia (Fig. 4 and Table III). The results may indicate a new therapeutic strategy for pancreatic cancer.

Survival prediction of the 6 ATO target genes in patients with pancreatic cancer. After the 6 ATO target genes associated with pancreatic cancer were identified, the K-M plotter was used to analyze the association of these genes with the overall survival of 177 patients with pancreatic cancer. As shown in Fig. 5, a low AKT1 level indicated poorer prognosis in patients with pancreatic cancer [hazard ratio $(\mathrm{HR})=0.60 ; \mathrm{P}=0.04]$. Furthermore, a high level of CCND1 was correlated with poor prognosis of patients with pancreatic cancer $(\mathrm{HR}=2.71$; $\left.\mathrm{P}=2 \times 10^{-4}\right)$. MAPK1 may also serve as a prognostic factor for patients with pancreatic cancer $(\mathrm{HR}=1.74 ; \mathrm{P}<0.01)$, with high expression levels indicating poor prognosis. However, CKDN2A $(\mathrm{P}=0.17)$, IKBKB $(\mathrm{P}=0.33)$ and MAPK3 $(\mathrm{P}=0.24)$ were not found to be prognostic factors for patients with pancreatic cancer.
Differential expression of the 3 ATO target genes in pancreatic cancer. As aforementioned, AKT1, CCND1 and MAPK1 were demonstrated to be associated with the prognosis of pancreatic cancer. Differential expression analysis of these three ATO target genes was then conducted using the Oncomine website. As shown in Fig. 6, AKT1 was observed to be overexpressed in pancreatic cancer compared with normal pancreatic tissue in the studies of Ishikawa et al (31), Iacobuzio-Donahue et al (32) and Pei et al (33) $(\mathrm{P}<0.01, \mathrm{P}<0.01$ and $<0.01$, respectively). Badea et al (34),Logdons et al (35) and Pei et al (33) also reported that CCND1 was more highly expressed in pancreatic cancer as compared with the normal pancreas $\left(\mathrm{P}=4.04 \times 10^{-6}, \mathrm{P}<0.01\right.$ and $\mathrm{P}=5.51 \times 10^{-4}$, respectively). Furthermore, MAPK1 was reported to be overexpressed in pancreatic cancer by Pei et al (33) and Segara et al (36) $(\mathrm{P}=0.01$ and $\mathrm{P}<0.01$, respectively).

\section{Discussion}

Pancreatic cancer is one of the most aggressive malignancies and the fourth cause of cancer-associated mortalities worldwide. This cancer is frequently diagnosed at an advanced stage, and most tumors are unresectable at clinical presentation (37), which leads to a poor prognosis and high mortality rates among patients with pancreatic cancer. Despite the advances in diagnostic and treatment methods, there have been no significant improvements in the prognosis of patients with pancreatic cancer in the past years. Therefore, more effective treatment methods for pancreatic cancer are urgently required.

The antitumor properties of ATO have been identified in a number of tumors (10-14). ATO has also been shown to inhibit the viability of pancreatic cancer stem cells in vitro 


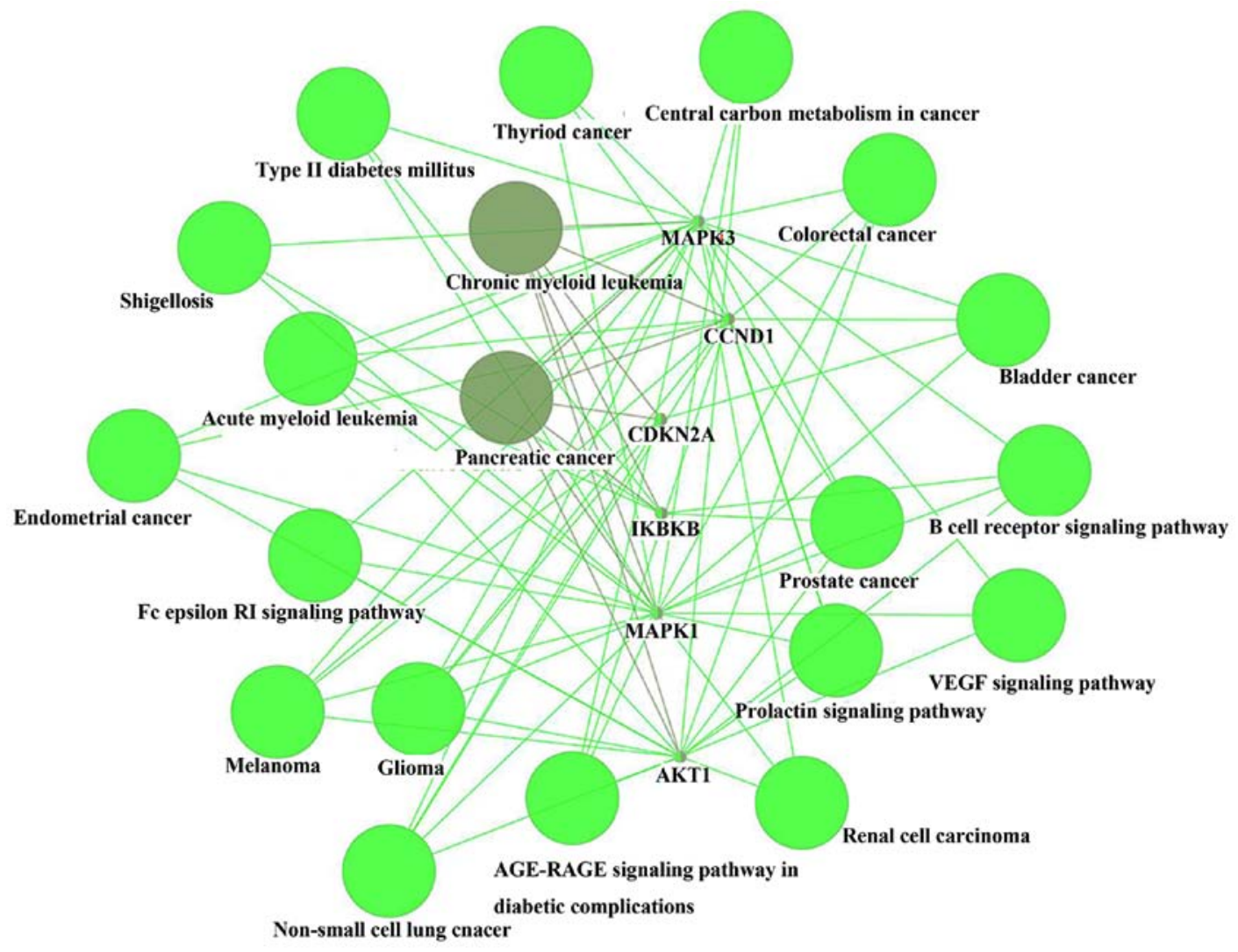

Figure 2. A visual display of Kyoto Encyclopedia of Genes and Genomes pathways in 6 arsenic trioxide target genes (AKT1, CCND1, CDKN2A, IKBKB, MAPK1 and MAPK3) associated with pancreatic cancer.
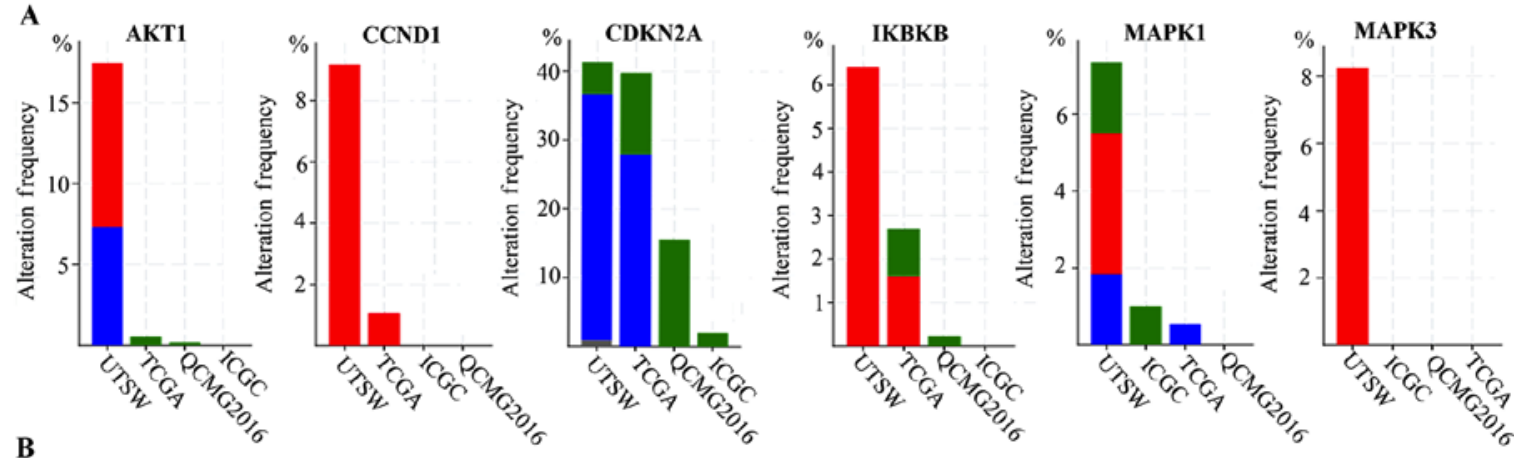

Study of origin

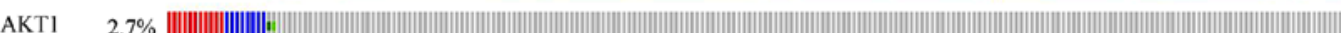

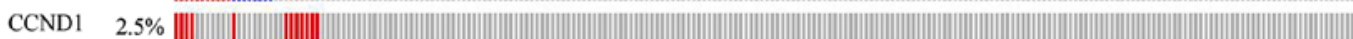

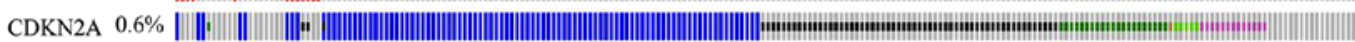

IKBKB $\quad 1.7 \%$ |

MAPK1 $\quad 1.3 \%$ |

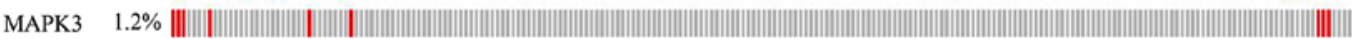

| Amplification | Deep deletion || Missense mutation | Truncating mutation || No alterations

Figure 3. Genetic alterations of arsenic trioxide target genes (AKT1, CCND1, CDKN2A, IKBKB, MAPK1 and MAPK3) in four pancreatic cancer studies, embedded in the cBioPortal for Cancer Genomics. ICGC, QCMG2016, TCGA and UTSW represent the different origin of the studies. (A) Overview of changes on genes (AKT1, CCND1, CDKN2A, IKBKB, MAPK1 and MAPK3) in genomics datasets available in four studies. (B) OncoPrint, providing a visual summary of alteration across a set of pancreatic cancer samples based on a query of the 6 genes. There were a total of 850 samples in the four studies included in the analysis.

and in vivo (38). In addition, Gao et al (15) reported that ATO exerted anticancer effects on pancreatic cancer. The combination of ATO with hypoxia-inducible factor-1 inhibitor was also found to be effective (39). However, the potential targets and molecular mechanisms of action of ATO in pancreatic cancer remain elusive. 
Table III. Kyoto Encyclopedia of Genes and Genomes pathways in the protein-protein interaction network of the arsenic trioxide target genes interacted 20 genes.

\begin{tabular}{|c|c|c|c|}
\hline Pathway & Count & False discovery rate & Gene name \\
\hline Hepatitis B & 13 & $4.42 \times 10^{-23}$ & $\begin{array}{l}\text { CDK4, CDK6, CDKN1A, CDKN1B, JUN, NFKB1, } \\
\text { PIK3CA, PIK3CB, PIK3CD, PIK3CG, PTEN, RB1, TP53 }\end{array}$ \\
\hline Prostate cancer & 12 & $4.42 \times 10^{-23}$ & $\begin{array}{l}\text { CDKN1A, CDKN1B, FOXO1, MTOR, NFKB1, PIK3CA, } \\
\text { PIK3CB, PIK3CD, PIK3CG, PTEN, RB1, TP53 }\end{array}$ \\
\hline Pathways in cancer & 15 & $4.43 \times 10^{-23}$ & $\begin{array}{l}\text { CDK4, CDK6, CDKN1A, CDKN1B, FOXO1, JUN, MTOR, } \\
\text { NFKB1, PIK3CA, PIK3CB, PIK3CD, PIK3CG, PTEN, } \\
\text { RB1, TP53 }\end{array}$ \\
\hline Glioma & 11 & $1.43 \times 10^{-22}$ & $\begin{array}{l}\text { CDK4, CDK6, CDKN1A, MTOR, PIK3CA, PIK3CB, } \\
\text { PIK3CD, PIK3CG, PTEN, RB1, TP53 }\end{array}$ \\
\hline Chronic myeloid leukemia & 11 & $4.99 \times 10^{-22}$ & $\begin{array}{l}\text { CDK4, CDK6, CDKN1A, CDKN1B, NFKB1, PIK3CA, } \\
\text { PIK3CB, PIK3CD, PIK3CG, RB1, TP53 }\end{array}$ \\
\hline Small cell lung cancer & 11 & $4.21 \times 10^{-21}$ & $\begin{array}{l}\text { CDK4, CDK6, CDKN1B, NFKB1, PIK3CA, PIK3CB, } \\
\text { PIK3CD, PIK3CG, PTEN, RB1, TP53 }\end{array}$ \\
\hline Viral carcinogenesis & 12 & $1.16 \times 10^{-19}$ & $\begin{array}{l}\text { CDK4, CDK6, CDKN1A, CDKN1B, JUN, NFKB1, } \\
\text { PIK3CA, PIK3CB, PIK3CD, PIK3CG, RB1, TP53 }\end{array}$ \\
\hline Melanoma & 10 & $1.48 \times 10^{-19}$ & $\begin{array}{l}\text { CDK4, CDK6, CDKN1A, PIK3CA, PIK3CB, PIK3CD, } \\
\text { PIK3CG, PTEN, RB1, TP53 }\end{array}$ \\
\hline PI3K-AKT signaling pathway & 13 & $1.27 \times 10^{-18}$ & $\begin{array}{l}\text { CDK4, CDK6, CDKN1A, CDKN1B, FOXO3, MTOR, } \\
\text { NFKB1, PIK3CA, PIK3CB, PIK3CD, PIK3CG, PTEN, } \\
\text { TP53 }\end{array}$ \\
\hline Non-small cell lung cancer & 9 & $4.22 \times 10^{-18}$ & $\begin{array}{l}\text { CDK4, CDK6, FOXO3, PIK3CA, PIK3CB, PIK3CD, } \\
\text { PIK3CG, RB1, TP53 }\end{array}$ \\
\hline
\end{tabular}

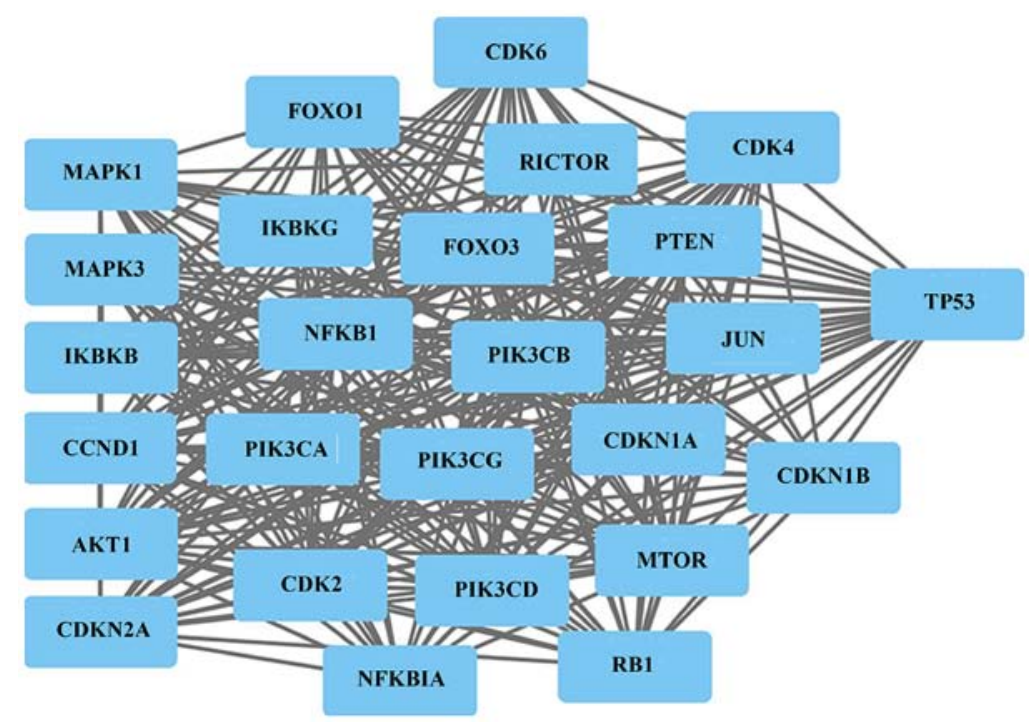

Figure 4. Visualization of the gene network connected to AKT1, CCND1, CDKN2A, IKBKB, MAPK1 and MAPK3.

In the present study, the molecular targets and associated genes of ATO were investigated by bioinformatics analysis. First, the 19 target genes of ATO were identified by DrugBank 5.0 and STITCH, which included IKBKB, TXNRD1, JUN, CCND1, MAPK3, MAPK1, AKT1, CDKN1A, HDAC1, PML, RARA, ESR1, CDKN2A, ABCC1, SP1, DNMT3B, DNMT1, HSPA4 and ABCB1. Next, the STRING website was used to construct the PPI network and conduct KEGG pathway analysis for the ATO target genes. A total of 6 target genes were demonstrated to be associated with pancreatic cancer, including AKT1, CCND1, CDKN2A, IKBKB, MAPK1 and MAPK3. Subsequently, the cBioPortal database was used to detect the alterations of these 6 genes, and the results were as follows: AKT1, IKBKB and MAPK1 were found to display amplification, deep deletion and 

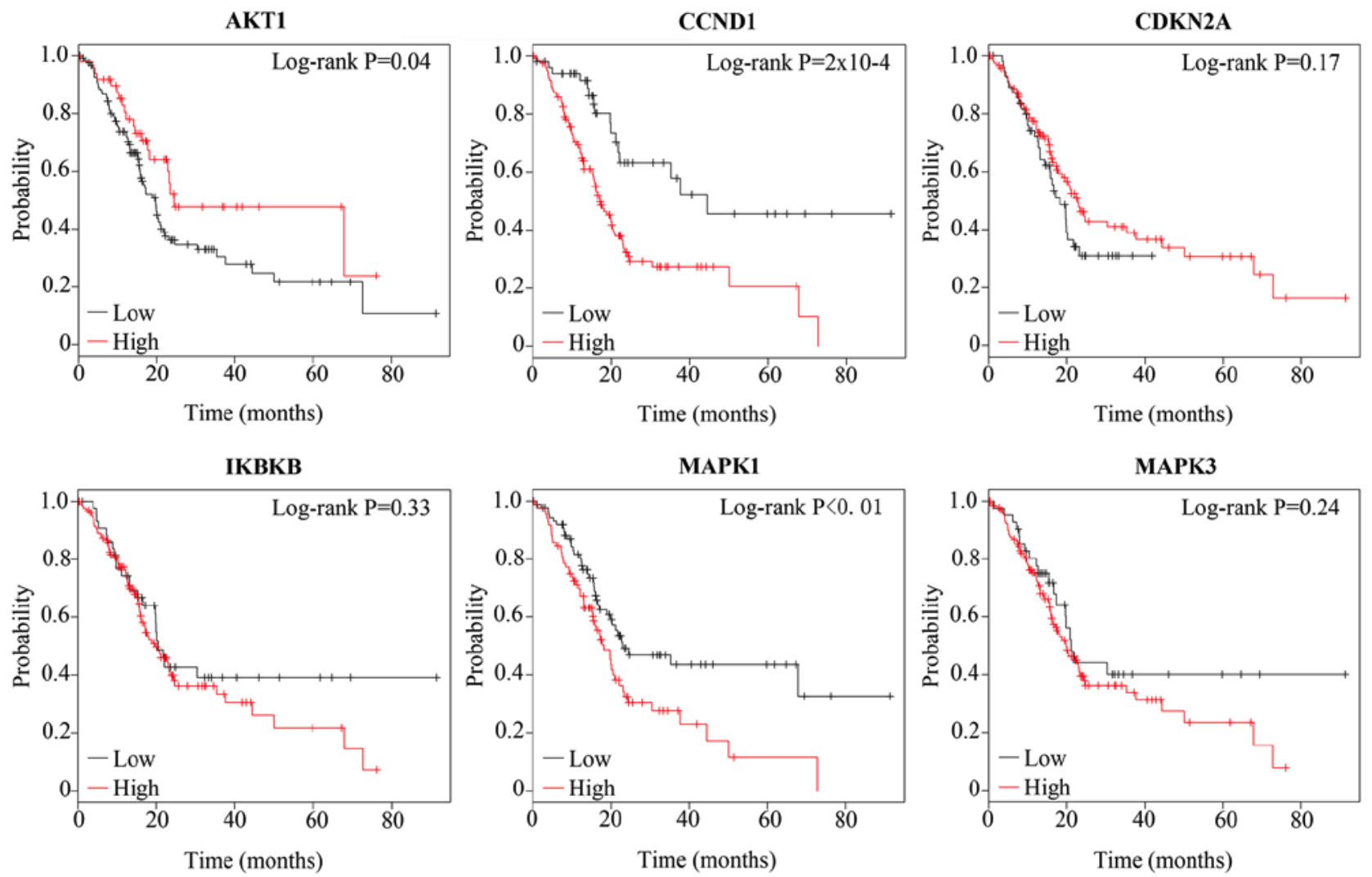

Figure 5. Prognostic analysis of arsenic trioxide target genes (AKT1, CCND1, CDKN2A, IKBKB, MAPK1 and MAPK3) in pancreatic cancer patients using the Kaplan-Meier plotter website. A total of 177 samples were used for each prognostic analysis.

missense mutations; CDKN2A exhibited deep deletion, truncating, missense, inframe and other mutations; and CCND1 and MAPK3 exhibited amplification. Furthermore, 20 genes that are interconnected with the 6 ATO target genes in pancreatic cancer were identified. These genes were mainly involved in hepatitis B, pancreatic cancer, pathways in cancer, glioma and chronic myeloid leukemia. Finally, the K-M plotter was used to analyze the association of the 6 target genes with the overall survival of patients with pancreatic cancer and Oncomine was used to analyze differential expression of these genes in pancreatic cancer. The results demonstrated that CCND1 and MAPK1 were overexpressed in pancreatic cancer and may be of prognostic value for patients with pancreatic cancer.

By conducting bioinformatics analysis, 6 ATO target genes were identified in the current study. Among these 6 genes, AKT1 is a member of the PI3K/AKT pathway and serves an important role in multiple types of cancer, including pancreatic cancer (40). CCND1, also referred to as cyclin D1, forms a complex with CDK4, thus phosphorylating and inhibiting RB1, and regulating the cell cycle during G1/S transition. The activation of the CCND1/CDK4 complex also promotes pancreatic cancer cell growth (41). Furthermore, it is well-established that the increased risk of pancreatic cancer is partly due to germline mutation carrier of CDKN2A (42). A previous study demonstrated that nuclear factor (NF) $-\kappa B$ promoted the proliferation and growth, and inhibited the apoptosis of pancreatic cancer cells (43). Inhibition of NF- $\kappa B$ also blocked metastasis in pancreatic cancer-derived xenograft tumors (44). In another study, the MAPK/ERK signaling pathway was revealed to be correlated with cancer cell growth, proliferation and apoptosis, including pancreatic cancer (45). Hu et al (46) used integrative analysis and identified MAPK3 as a significant hub gene in pancreatic cancer. In the present study, AKT1, CCND1 and MAPK1 were found to be overexpressed in pancreatic cancer, while high CCND1 and MAPK1 levels indicated poor prognosis.

STRING analysis performed in the current study revealed that the 6 ATO target genes (AKT1, CCND1, CDKN2A, IKBKB, MAPK1 and MAPK3) were involved in chronic myeloid leukemia, pancreatic cancer, non-small cell lung cancer, acute myeloid leukemia, melanoma, glioma, prostate cancer, endocine resistance FoxO signaling and hepatitis B. The PPI network was also used to analyze the genes interconnected with the 6 ATO target genes, and the 20 interconnected genes were observed to be involved in hepatitis B, prostate cancer, pathways in cancer, glioma and chronic myeloid leukemia. These results may help develop new therapeutic strategies for cancer.

However, there were certain limitations to the present study. Firstly, this research was based on bioinformatics analysis, and experimental results are required to support the conclusions. Secondly, since the NF- $\mathrm{kB}$ pathway serves an important role in multiple cancer development, a significant association of IKBKB with poor prognosis of patients with pancreatic cancer was not observed by Kaplan-Meier analysis. Furthermore, the key genes implicated in pancreatic cancer should also be analyzed to confirm the reliability of the results. The present study showed that 6 ATO target genes (AKT1, CCND1, CDKN2A, IKBKB, MAPK1 and MAPK3) were associated 

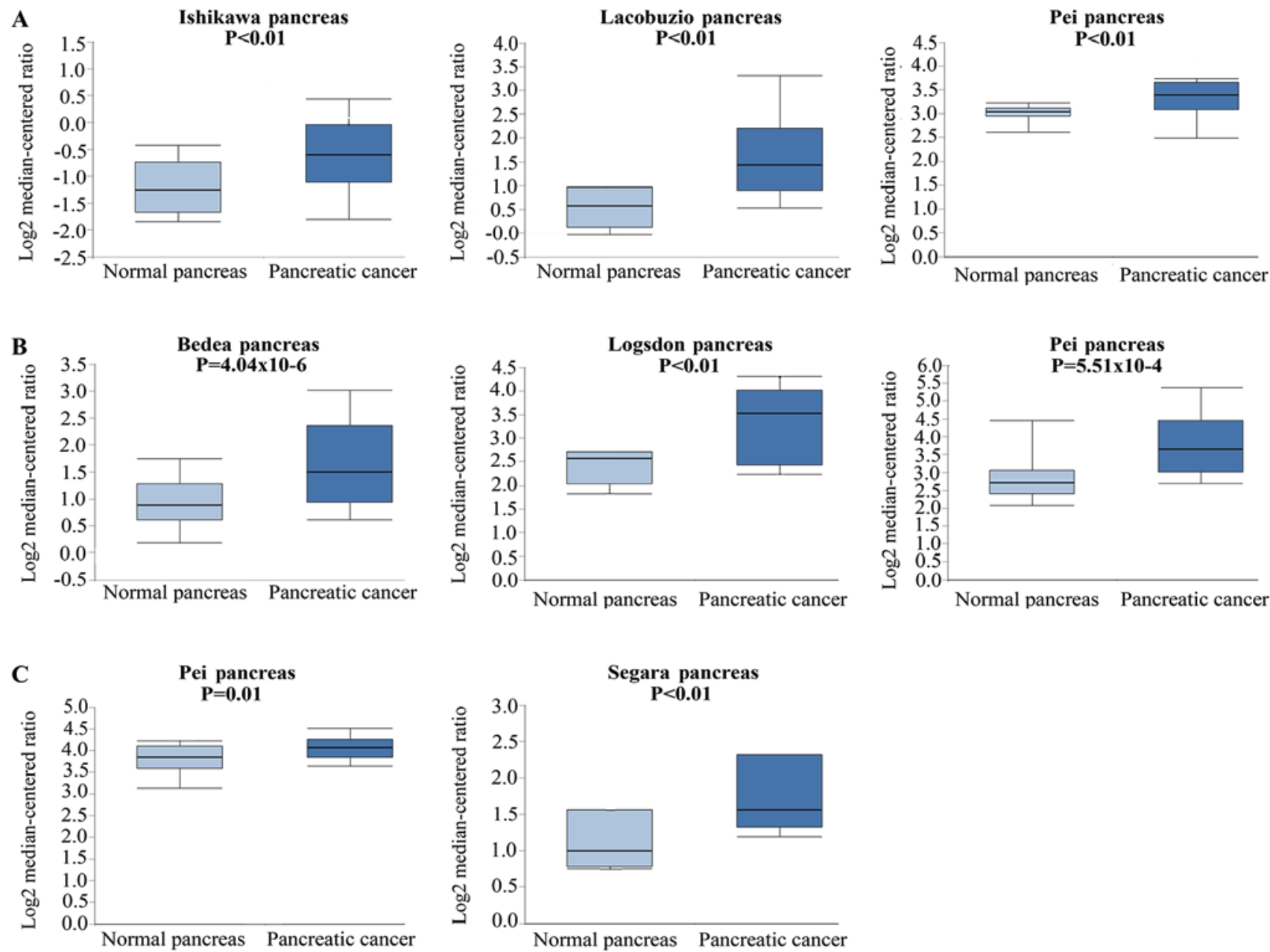

Figure 6. Analysis of the expression of AKT1, CCND1 and MAPK1 in pancreatic cancer and normal pancreatic tissues, performed with the Oncomine website. The expression of (A) AKT1, (B) CCND1 and (C) MAPK1 in different pancreatic cancer studies is shown.

with pancreatic cancer, and that high CCND1 and MAPK1 levels indicated poor prognosis. However, since tissue samples were not collected, these 6 proteins could not be verified in pancreatic cancer samples. Next, immunohistochemistry will be used to validate the expression of these proteins in pancreatic cancer and evaluate their association with the prognosis of patients with pancreatic cancer.

In conclusion, the bioinformatics analysis was used to elucidate the molecular mechanism of action of ATO in pancreatic cancer. The results not only suggest that ATO may be an effective drug for pancreatic cancer, but also provide a novel approach for identifying potential treatments for other diseases by bioinformatics analysis.

\section{Acknowledgements}

Not applicable.

\section{Funding}

The present study was supported by grants from the National Natural Science Foundation of China (no. 81672921) and the Innovation Capacity Support Plan of Shaanxi Province (no. 2018TD-002).

\section{Availability of data and materials}

The datasets generated and analyzed during the present study are available in the public databases.

\section{Authors' contributions}

QZ and SXH designed the study. CYZ acquired and analyzed the data, drafted the manuscript and revised it. LYG was involved in data analysis and revised the manuscript. RL, NNW, YYF, YPD, HZ, YQZ and YYZ performed the data analysis. All authors read and approved the final manuscript.

\section{Ethics approval and consent to participate}

Not applicable.

\section{Patient consent for publication}

Not applicable.

\section{Competing interests}

The authors declare that they have no competing interests. 


\section{References}

1. Siegel RL, Miller KD and Jemal A: Cancer statistics, 2017. CA Cancer J Clin 67: 7-30, 2017.

2. Saad AM, Turk T, Al-Husseini MJ and Abdel-Rahman O: Trends in pancreatic adenocarcinoma incidence and mortality in the United States in the last four decades; a SEER-based study. BMC Cancer 18: 688, 2018.

3. Siegel RL, Miller KD and Jemal A: Cancer statistics, 2018. CA Cancer J Clin 68: 7-30, 2018.

4. Soignet SL, Frankel SR, Douer D, Tallman MS, Kantarjian H, Calleja E, Stone RM, Kalaycio M, Scheinberg DA, Steinherz P, et al: United States multicenter study of arsenic trioxide in relapsed acute promyelocytic leukemia. J Clin Oncol 19: 3852-3860, 2001

5. Mathews V, George B, Chendamarai E, Lakshmi KM, Desire S, Balasubramanian P, Viswabandya A, Thirugnanam R, Abraham A, Shaji RV, et al: Single-agent arsenic trioxide in the treatment of newly diagnosed acute promyelocytic leukemia: Long-term follow-up data. J Clin Oncol 28: 3866-3871, 2010.

6. Ghavamzadeh A, Alimoghaddam K, Rostami S, Ghaffari SH, Jahani M, Iravani M, Mousavi SA, Bahar B and Jalili M: Phase II study of single-agent arsenic trioxide for the front-line therapy of acute promyelocytic leukemia. J Clin Oncol 29: 2753-2757, 2011.

7. Lo-Coco F, Avvisati G, Vignetti M, Thiede C, Orlando SM, Iacobelli S, Ferrara F, Fazi P, Cicconi L, Di Bona E, et al: Retinoic acid and arsenic trioxide for acute promyelocytic leukemia. N Engl J Med 369: 111-121, 2013.

8. Burnett AK, Russell NH, Hills RK, Bowen D, Kell J, Knapper S, Morgan YG, Lok J, Grech A, Jones G, et al: Arsenic trioxide and all-trans retinoic acid treatment for acute promyelocytic leukaemia in all risk groups (AML17): Results of a randomised, controlled, phase 3 trial. Lancet Oncol 16 1295-1305, 2015.

9. Seftel MD, Barnett MJ, Couban S, Leber B, Storring J, Assaily W, Fuerth B, Christofides A and Schuh AC: A Canadian consensus on the management of newly diagnosed and relapsed acute promyelocytic leukemia in adults. Curr Oncol 21: 234-250, 2014.

10. Zhang L, Liu L, Zhan S, Chen L, Wang Y, Zhang Y, Du J, Wu Y and Gu L: Arsenic trioxide suppressed migration and angiogenesis by targeting FOXO3a in gastric cancer cells. Int J Mol Sci 19: pii: E3739, 2018.

11. Zhong L, Xu F and Chen F: Arsenic trioxide induces the apoptosis and decreases NF-KB expression in lymphoma cell lines. Oncol Lett 16: 6267-6274, 2018.

12. Mao MH, Huang HB, Zhang XL, Li K, Liu YL and Wang P. Additive antitumor effect of arsenic trioxide combined with intravesical bacillus Calmette-Guerin immunotherapy against bladder cancer through blockade of the IER3/Nrf2 pathway. Biomed Pharmacother 107: 1093-1103, 2018.

13. Du S, Liu K, Gao P, Li Z and Zheng J: Differential anticancer activities of arsenic trioxide on head and neck cancer cells with different human papillomavirus status. Life Sci 212: 182-193, 2018.

14. Luo D, Zhang X, Du R, Gao W, Luo N, Zhao S, Li Y, Chen R, Wang H, Bao Y, et al: Low dosage of arsenic trioxide $\left(\mathrm{As}_{2} \mathrm{O}_{3}\right)$ inhibits angiogenesis in epithelial ovarian cancer without cell apoptosis. J Biol Inorg Chem 23: 939-947, 2018.

15. Gao JK, Wang LX, Long B, Ye XT, Su JN, Yin XY, Zhou XX and Wang ZW: Arsenic Trioxide inhibits cell growth and invasion via Down-Regulation of Skp2 in pancreatic cancer cells. Asian Pac J Cancer Prev 16: 3805-3810, 2015.

16. Huang YA, You ZH and Chen X: A systematic prediction of drug-target interactions using molecular fingerprints and protein sequences. Curr Protein Pept Sci 19: 468-478, 2018.

17. Wishart DS, Knox C, Guo AC, Cheng D, Shrivastava S, Tzur D, Gautam B and Hassanali M: DrugBank: A knowledgebase for drugs, drug actions and drug targets. Nucleic Acids Res 36: D901-D906, 2008.

18. Kuhn M, von Mering C, Campillos M, Jensen LJ and Bork P: STITCH: Interaction networks of chemicals and proteins. Nucleic Acids Res 36: D684-D688, 2008.

19. Szklarczyk D, Franceschini A, Wyder S, Forslund K, Heller D, Huerta-Cepas J, Simonovic M, Roth A, Santos A, Tsafou KP, et al: STRING v10: Protein-protein interaction networks, integrated over the tree of life. Nucleic Acids Res 43: D447-D452, 2015.
20. Szklarczyk D, Morris JH, Cook H, Kuhn M, Wyder S, Simonovic M, Santos A, Doncheva NT, Roth A, Bork P, et al: The STRING database in 2017: Quality-controlled protein-protein association networks, made broadly accessible. Nucleic Acids Res 45: D362-D368, 2017.

21. Shannon P, Markiel A, Ozier O, Baliga NS, Wang JT, Ramage D, Amin N, Schwikowski B and Ideker T: Cytoscape: A software environment for integrated models of biomolecular interaction networks. Genome Res 13: 2498-2504, 2003.

22. Bindea G, Galon J and Mlecnik B: CluePedia Cytoscape plugin: Pathway insights using integrated experimental and in silico data. Bioinformatics 29: 661-663, 2013.

23. Cerami E, Gao J, Dogrusoz U, Gross BE, Sumer SO, Aksoy BA, Jacobsen A, Byrne CJ, Heuer ML, Larsson E, et al: The cBio cancer genomics portal: An open platform for exploring multidimensional cancer genomics data. Cancer Discov 2: 401-404, 2012.

24. Gao J, Aksoy BA, Dogrusoz U, Dresdner G, Gross B, Sumer SO, Sun Y, Jacobsen A, Sinha R, Larsson E, et al: Integrative analysis of complex cancer genomics and clinical profiles using the cBioPortal. Sci Signal 6: pl1, 2013.

25. Biankin AV, Waddell N, Kassahn KS, Gingras MC, Muthuswamy LB, Johns AL, Miller DK, Wilson PJ, Patch AM, $\mathrm{Wu}$ J, et al: Pancreatic cancer genomes reveal aberrations in axon guidance pathway genes. Nature 491: 399-405, 2012.

26. Bailey P, Chang DK, Nones K, Johns AL, Patch AM, Gingras MC Miller DK, Christ AN, Bruxner TJ, Quinn MC, et al: Genomic analyses identify molecular subtypes of pancreatic cancer. Nature 531: 47-52, 2016.

27. Sanchez-Vega F, Mina M, Armenia J, Chatila WK, Luna A, La KC, Dimitriadoy S, Liu DL, Kantheti HS, Saghafinia S, et al: Oncogenic signaling pathways in the cancer genome atlas. Cell 173: 321-337.e10, 2018.

28. Witkiewicz AK, McMillan EA, Balaji U, Baek G, Lin WC, Mansour J, Mollaee M, Wagner KU, Koduru P, Yopp A, et al: Whole-exome sequencing of pancreatic cancer defines genetic diversity and therapeutic targets. Nat Commun 6: 6744, 2015.

29. Lanczky A, Nagy A, Bottai G, Munkácsy G, Szabó A, Santarpia L and Gyórffy B: miRpower: A web-tool to validate survival-associated miRNAs utilizing expression data from 2178 breast cancer patients. Breast Cancer Res Treat 160: 439-446, 2016.

30. Rhodes DR, Kalyana-Sundaram S, Mahavisno V, Varambally R, Yu J, Briggs BB, Barrette TR, Anstet MJ, Kincead-Beal C, Kulkarni P, et al: Oncomine 3.0: Genes, pathways, and networks in a collection of 18,000 cancer gene expression profiles. Neoplasia 9: 166-180, 2007

31. Ishikawa M, Yoshida K, Yamashita Y, Ota J, Takada S, Kisanuki H, Koinuma K, Choi YL, Kaneda R, Iwao T, et al: Experimental trial for diagnosis of pancreatic ductal carcinoma based on gene expression profiles of pancreatic ductal cells. Cancer Sci 96: 387-393, 2005.

32. Iacobuzio-Donahue CA, Maitra A, Olsen M, Lowe AW, van Heek NT, Rosty C, Walter K, Sato N, Parker A, Ashfaq R, et al: Exploration of global gene expression patterns in pancreatic adenocarcinoma using cDNA microarrays. Am J Pathol 162: 1151-1162, 2003.

33. Pei H, Li L, Fridley BL, Jenkins GD, Kalari KR, Lingle W, Petersen G, Lou Z and Wang L: FKBP51 affects cancer cell response to chemotherapy by negatively regulating Akt. Cancer Cell 16: 259-266, 2009.

34. Badea L, Herlea V, Dima SO, Dumitrascu T and Popescu I: Combined gene expression analysis of whole-tissue and microdissected pancreatic ductal adenocarcinoma identifies genes specifically overexpressed in tumor epithelia. Hepatogastroenterology 55: 2016-2027, 2008.

35. Logsdon CD, Simeone DM, Binkley C, Arumugam $T$, Greenson JK, Giordano TJ, Misek DE, Kuick R and Hanash S: Molecular profiling of pancreatic adenocarcinoma and chronic pancreatitis identifies multiple genes differentially regulated in pancreatic cancer. Cancer Res 63: 2649-2657, 2003.

36. Segara D, Biankin AV, Kench JG, Langusch CC, Dawson AC, Skalicky DA, Gotley DC, Coleman MJ, Sutherland RL and Henshall SM: Expression of HOXB2, a retinoic acid signaling target in pancreatic cancer and pancreatic intraepithelial neoplasia. Clin Cancer Res 11: 3587-3596, 2005.

37. Chari ST: Detecting early pancreatic cancer: Problems and prospects. Semin Oncol 34: 284-294, 2007.

38. Han JB, Sang F, Chang JJ, Hua YQ, Shi WD, Tang LH and Liu LM: Arsenic trioxide inhibits viability of pancreatic cancer stem cells in culture and in a xenograft model via binding to SHH-Gli. Onco Targets Ther 6: 1129-1138, 2013 
39. Lang M, Wang X, Wang H, Dong J, Lan C, Hao J, Huang C, Li X, Yu M, Yang Y, et al: Arsenic trioxide plus PX-478 achieves effective treatment in pancreatic ductal adenocarcinoma. Cancer Lett 378: 87-96, 2016.

40. Michl P and Downward J: Mechanisms of disease: PI3K/AKT signaling in gastrointestinal cancers. Z Gastroenterol 43: 1133-1139, 2005.

41. Yan L, Wang Y, Wang ZZ, Rong YT, Chen LL, Li Q, Liu T, Chen YH, Li YD, Huang ZH and Peng J: Cell motility and spreading promoted by CEACAM6 through cyclin D1/CDK4 in human pancreatic carcinoma. Oncol Rep 35: 418-426, 2016.

42. McWilliams RR, Wieben ED, Chaffee KG, Antwi SO, Raskin L, Olopade OI, Li D, Highsmith WE Jr, Colon-Otero G, Khanna LG, et al: CDKN2A Germline rare coding variants and risk of pancreatic cancer in minority populations. Cancer Epidemiol Biomarkers Prev 27: 1364-1370, 2018.

43. Liptay S, Weber CK, Ludwig L, Wagner M, Adler G and Schmid RM: Mitogenic and antiapoptotic role of constitutive NF-kappaB/Rel activity in pancreatic cancer. Int J Cancer 105: 735-746, 2003.
44. Fujioka S, Sclabas GM, Schmidt C, Frederick WA, Dong QG, Abbruzzese JL, Evans DB, Baker C and Chiao PJ: Function of nuclear factor kappaB in pancreatic cancer metastasis. Clin Cancer Res 9: 346-354, 2003.

45. Hu Y, Yang H, Lu XQ, Xu F, Li J and Qian J: ARHI suppresses pancreatic cancer by regulating MAPK/ERK 1/2 pathway. Pancreas 44: 342-343, 2015.

46. Hu B, Shi C, Jiang HX and Qin SY: Identification of novel therapeutic target genes and pathway in pancreatic cancer by integrative analysis. Medicine (Baltimore) 96: e8261, 2017.

This work is licensed under a Creative Commons Attribution-NonCommercial-NoDerivatives 4.0 International (CC BY-NC-ND 4.0) License. 